\title{
Cancer Vaccination, Will You Have To Pay The Toll?
}

\author{
Sean M. Gregory ${ }^{1,2}$, John A. West ${ }^{1,2}$ and Blossom Damania ${ }^{1,2,3 *}$
}

${ }^{1}$ Lineberger Comprehensive Cancer Center, University of North Carolina at Chapel Hill, Chapel Hill, NC27599, USA

${ }^{2}$ Department of Microbiology \& Immunology, University of North Carolina at Chapel Hill, Chapel Hill, NC27599, USA

${ }^{3}$ UNC Center for AIDS Research, University of North Carolina at Chapel Hill, Chapel Hill, NC 27599, USA

\begin{abstract}
Cancer vaccines based on patient-derived, autologous immune cells are actively being pursued as a novel strategy to utilize the body's natural defenses against malignancy. Harnessing the ability of the immune system to fight cancer involves overcoming many obstacles including tumor-specific targeting, overcoming tolerance, and generating effective tumoricidal responses. Co-administration of immune activating adjuvants may hold the key to breaking through several of these barriers. Toll-like receptors (TLR) are pathogen sensors of the innate immune system that activate proinflammatory responses to fight infection and initiate adaptive immune responses. TLRs are increasingly being explored in combination with cancer vaccine strategies since they may have the potential to enhance immunotherapy by promoting tumor-specific immunity. This review will focus on recent basic and clinical research on the use of TLR agonists in cancer therapy.
\end{abstract}

Keywords: Cancer vaccines; TLR; Innate immunity; Dendritic cells

\section{Introduction}

The immune system can function as an active tumor suppressor mechanism because tumors are more likely to arise in an immune compromised host versus immune competent [1]. In addition, it is widely accepted that tumor-associated antigens (TAA) can be targeted by the immune system since they can be used to raise tumor-specific antibodies or cytotoxic T cells. TAAs are otherwise normal cellular antigens that have been altered in a way during tumorigenesis such that their expression level or sequence is changed as compared to the antigen expressed on healthy cells. For example, TAAs can be highly expressed or mutated in tumor cells compared to normal cells, and as a result are immunogenic. Overexpressed antigens include Her-2 found in breast carcinoma and MUC-1 and Wilm's tumor-1 (WT-1) found in several malignancies. Altered or mutated proteins include the key cellular signaling molecule Ras, and the well-known BCR-Abl mutation in chronic myeloid leukemia. This approach is the basis for an active area of cancer research, called immune therapy, in which the immune system is harnessed to fight cancer. However, even in light of recent successes in cancer vaccination, patient survival has only marginally increased, leaving open many questions regarding how to improve treatment protocols. It is clear that passive immunotherapybased cellular vaccines generated ex vivo exhibit limited efficacy and are extremely costly. The best option may be to enhance existing cancerspecific immunity in vivo (otherwise known as active immunotherapy). For example, proposed strategies include co-administration of potent adjuvants with the cancer vaccine, inhibiting immune negative regulation mechanisms to overcome tolerance, and/or exploring combinational therapies such as vaccination and chemotherapeutic regimens. Another approach gaining momentum involves activating innate immune molecules called Toll-like receptors (TLRs) using cognate agonists in combination with cancer vaccines to positively regulate the immune response towards tumor-specific immunity [2]. This review will discuss the therapeutic benefit of TLR agonists in cancer vaccination by discussing putative roles for enhancing efficacy through stimulation of innate and adaptive immune responses.

\section{History of Cancer Vaccines}

Immune cancer therapy with monoclonal antibodies is well established and effective, with therapeutic antibodies Trastuzumab
(Herceptin), Rituximab (Rituxan) and Cetuximab (Erbitux) being the best examples. Trastuzumab targets and blocks the HER2 transmembrane receptor and its intracellular signaling cascade. Since the HER2 gene is amplified in many breast cancer patients, treating the disease by inhibiting the signaling of the overexpressed HER2 protein results in significant clinical responses. This approach is also the basis for Rituximab and Cetuximab, which target cellular proteins CD20 and epidermal growth factor receptor (EGFR), respectively. Another approach involving adoptive transfer of autologous $\mathrm{T}$ cells that have been raised ex vivo against whole tumor cells or a TAA usually loaded onto dendritic cells was the first indication that cellular therapy may be a viable way to eradicate cancer [3]. Adoptive transfer was originally used against solid tumors and Epstein-Barr virus lymphoma [4]. However, there remains the possibility for tumor recurrence [5]. Just as vaccination against a virus must confer long-term protection to yield maximum efficacy, so too must a cancer vaccine. In order to establish cancer immunity, immune cells must be capable of recognizing the tumor, eradicating it and generating memory cells for future immunemediated cancer targeting in the event of a relapse.

Cancer vaccines have been tested for a number of malignancies including advanced melanoma, breast, pancreatic and prostate cancers. While initial trials mainly focused on safety, immune responses to tumor antigens were observed, suggesting that active immunization against tumors could be achieved. Several clinical trials have confirmed this, albeit with mixed results. BiovaxID (Biovest International, INC), a follicular-lymphoma (FL) idiotype-derived (Id) antigen conjugated to keyhole limpet hemocyanin (KLH) therapeutic vaccine with granulocyte macrophage-colony stimulating factor (GM-CSF), showed

*Corresponding author: Blossom Damania, Lineberger Comprehensive Cancer Center, University of North Carolina, Chapel Hill, NC27599, USA, Tel: (919) 843 6011; Fax: (919) 966ᄀ9673; E-mail: damania@med.unc.edu

Received September 10, 2011; Accepted November 06, 2011; Published November 10, 2011

Citation: Gregory SM, West JA, Damania B (2011) Cancer Vaccination, Will You Have To Pay The Toll? J Vaccines Vaccin S1:001. doi:10.4172/2157-7560.S1-001

Copyright: (c) 2011 Gregory SM, et al. This is an open-access article distributed under the terms of the Creative Commons Attribution License, which permits unrestricted use, distribution, and reproduction in any medium, provided the original author and source are credited. 
significant lymphoma-specific CD8+ cytotoxic T lymphocyte (CTL) responses in combination with chemotherapy in a phase II trial, which correlated with some tumor remission [6,7]. Recently, a phase III trial of treatment-naïve patients with advanced stage FL using BiovaxID in combination with KLH adjuvant and GM-CSF demonstrated that patients vaccinated against Id showed a disease-free survival of 44.2 months versus 30.6 months for the control. This is contrasted with two phase III Id-vaccines, Genitope and Favrille, which did not show increased clinical benefit. BiovaxID has also been tested in a phase II trial against Mantle Cell lymphoma in patients depleted of B cells [8]. The results of this study suggested that even in the absence of B cells, tumor specific immune responses could be.

Sipuleucel-T (Provenge) is the first FDA approved cellular immunotherapy for the treatment of asymptomatic or minimally symptomatic metastatic castration-resistant prostate cancer. In a large, double-blind phase III trial, patients who received APCs pulsed with prostatic acid phosphatase (PAA) and GM-CSF showed an increased median survival of approximately four months $[9,10]$. This modest increase in overall survival is a major advance for a disease that has a poor prognosis and therapeutically is very difficult to treat. Another prostate cancer immunotherapeutic is PROSTVAC, which is based on recombinant vaccinia and fowlpox poxvirus platforms encoding prostate specific antigen (PSA) and costimulatory moleculeslymphocyte function-associated antigen 3 (LFA-3), intercellular adhesion molecule 1 (ICAM-1) and B7-1 (CD80) [11]. Altogether, PROSTVAC is designed to elicit PSA-specific immunity through virus-dependent immune activation, and PSA antigen presentation. PROSTVAC treatment resulted in a $43 \%$ reduction in death and 8.5 month increase in median survival compared to the control arm [11]. Despite clinical success, these therapies may have limited long-term efficacy since they are designed to stimulate immune responses to only one antigen, and both PAA and PSA are normal self antigens that may present autoimmune complications in healthy tissue. Targeting multiple self antigens by vaccinating with whole tumor cells may be a better strategy since it would present additional potential immunogenic epitopes. GVAX, which is composed of prostate cancer cell lines LNCaP and PC3 that express GM-CSF, was developed to treat asymptomatic, castration-resistant prostate cancer. Unfortunately, two large phase III trials resulted in failure [12]. However, BioSante, in collaboration with Johns Hopkins University, reinitiated clinical research into GVAX by announcing a new phase II trial (NCT01417000), after preclinical data showed encouraging results when used in combination with the TLR4 agonist lipopolysaccharide [13]. The current study is designed to include GVAX along with cyclophosphamide to block regulatory T cells and Listeria monocytogenes to elicit stronger innate and adaptive immune responses, which may be critical for successful cancer vaccination.

\section{Significant Gaps in Cancer Vaccination}

While many different strategies have been employed to enhance antigen presenting cells (APC) e.g. dendritic cells, or cytotoxic activity of tumor-specific $\mathrm{T}$ cells elicited during vaccination regardless of whether they were expanded ex vivo or in vivo, significant challenges remain. Major hurdles involve enhancing $\mathrm{T}$ cell responses, increasing the duration of those responses, and development of long-term tumorspecific memory cells. In addition, efficient recruitment of $\mathrm{T}$ helper 1 (Th1) and APCs that have been adequately activated to upregulate costimulatory molecules must be achieved. Finally, immunological tolerance must be broken in instances where TAAs are targeted, which will likely be accomplished by inhibiting tumor-associated regulatory
T cells (Tregs) and myeloid-derived suppressor cells (MDSC). Thus, the appropriate adjuvant will likely have to be co-administered with any cancer vaccination strategy. Integration of agonists of an innate immune response with cancer vaccines may enhance efficacy due to their ability to improve uptake and antigen presentation, recruit inflammatory cells, prime the adaptive immune response, and possibly break immunological tolerance. TLRs are ubiquitous pathogen sensors of the innate immune system whose ligands have been incorporated into several recent vaccination strategies as adjuvants capable of enhancing cancer vaccine immunotherapy.

\section{Harnessing TLR Ligands for Cancer Vaccines}

The immune system is composed of an immediate innate immune response, usually occurring within a few hours after infection, followed by the adaptive immune response, which may take up to two weeks to achieve full strength. The adaptive immune response generally recognizes non-self antigens associated with a pathogen infection after they are presented on the surface of an APC. Pathogens that either infect APCs or are internalized by them activate the innate arm of the immune response by stimulating pattern-recognition receptors (PRRs). PRRs are germline-encoded receptors highly expressed in APCs, but found throughout the body, and capable of upregulating expression of anti-pathogen proinflammatory cytokines and driving the initiation of the adaptive arm of the host immune response. TLRs are a family of transmembrane PRRs ubiquitously expressed in APCs and inflammatory cells of the immune system [14]. They respond to pathogen-associated molecular patterns (PAMP) such as viral nucleic acid or bacterial cell wall constituents [14]. Stimulation of PRRs using adjuvants are a necessary part of successful pathogen vaccination strategies due to their ability to aid in antigen-specific immunity by promoting effector CTL and Th1 cell activation and long-term memory cell development [15].

TLRs have been investigated for their ability to generate strong immune responses to treat cancer, particularly metastatic melanoma and various carcinomas [16-19]. Moreover, TLR stimulation as an adjuvant may promote several key immunological events necessary for successful cancer vaccination in situations where the vaccine itself does not generate an effective immune response. For instance, upregulation of costimulatory molecules CD80 and CD86 on the surface of APCs is important for CTL activation. TLR stimulation also shifts the cytokine response to produce IL-12 from DCs and enhance the generation of CD4+ Th1 polarized T cells [20,21]. Th-1 cells are important for the secretion of effector cytokines like interferon- $\gamma$ and the T-cell proliferative cytokine, IL-2 [22].

TLR agonists can kill tumor cells when directly administered. For example, melanoma cells express TLR3 and TLR3 agonists induce apoptosis through activation of caspases [23]. Similar reports for other types of cancer cells with TLR3 ligand have been reported [24]. Clearly, in order for TLR agonists to work directly on tumors cells, it is imperative that the cancer cells express the cognate TLR. TLRs may also promote apoptosis, vascular permeability, lymphocyte homing to the tumor site and improve the sensitivity of the tumor to proapoptotic cytokines [24-26].

TAAs are capable of eliciting an immune response through the recruitment of APCs such as dendritic cells (DC), which take up the TAA and present it to tumor infiltrating CD8+ CTLs and also secrete the CD4+ Th1 promoting cytokine IL-12. Th1 cells secrete the proinflammatory cytokine interferon- $\gamma$ and IL- 2 to promote activation of CD8+ T cells and create an unfavorable environment for the tumor. 
However, tumor cells may secrete immunosuppressive factors of their own such as transforming-growth factor $\beta$ (TGF- $\beta$ ), indolamine2,3-dioxygenease (IDO) and fas ligand that are capable of recruiting anti-inflammatory CD4+ Th2 T cells, Tregs and MDSCs. Despite the immunogenic potential of cancer, immune suppression is often observed within the tumor microenvironment itself, thereby enabling favorable growth conditions for the tumor $[27,28]$. The presence of negative regulators of tumor-specific immune responses eventually takes over in the tumor microenvironment and generally by the time therapy is initiated, the balance is heavily in favor of the tumor. The immune suppressive environment in which the tumor resides could be counteracted by the administration of an appropriate adjuvant in combination with cancer vaccines [29].

Several cancer models have shown significant efficacy with TLR agonists in combination with immunotherapy protocols, and recent clinical trial data suggests this approach may hold the key to breaking immunological tolerance necessary for tumor-specific immune responses. TAAs are limited in their immunogenicity since the immune system is tolerant of these antigens. Therefore, breaking tolerance against TAAs may be the key to generating effective cancer vaccines and TLR activation may be important in this process [30,32]. TLR stimulation, in combination with vaccination with APCs loaded with TAAs, has been shown to break tolerance leading to enhanced vaccine efficacy and clinical responses.

\section{Evidence for the role of TLRs in cancer vaccine efficacy}

One approach to inducing immunity to TAAs involves direct immunization with messenger RNA (mRNA) encoding the TAA itself. In theory, the TAA mRNA should serve as an agonist for the induction of innate immunity through TLR7 ligation and antigenspecific adaptive immune responses. Using E.G7-OVA tumor model, direct vaccination with $\mathrm{mRNA}$ encoding OVA or prostate carcinomaassociated antigen, PSMA, induced antitumor responses in vivo with IL-12 levels significantly increased, which suggests this strategy promotes a favorable environment for Th1-dependent cell activation [33]. Although prophylactic administration of mRNA vaccination was not demonstrated the authors did suggest such experiments may be possible. Currently, phase I/II trials are underway in patients with hormone refractory prostate cancer (NCT00831467 and NCT00906243).

Long-term immunity associated with donor-lymphocyte infusion (DLI) for the treatment of chronic myelogenous leukemia (CML) is hypothesized to be a result of adjuvant effects of nucleic-acid antibody complexes in plasma [34]. TLRs 8 and 9 were required for the strong adjuvant effects of circulating antigen-antibody complexes with bound endogenous nucleic acids, which in the absence of DLI were not observed and rendered the DLI ineffective. This suggests that the therapeutic efficacy of DLI may stem from TLR-dependent activation to break tolerance towards CML-associated antigen. It will be interesting to determine in future work whether this is specific for $\mathrm{CML}$ or applicable to other malignant hematologic diseases.

In an effort to determine effective inducers of Th1 polarization and enhanced DC function, immunization with DCs treated with heparinbinding hemagglutinin (HBHA), a constituent of Mycobacterium tuberculosis, and pulsed with OVA peptide ex vivo resulted in decreased tumor growth and increased survival in a murine E.G7 thymoma model [35]. HBHA acts by stimulating TLR4 and upregulating the expression of costimulatory molecules, major histocompatibility complexes I and II, and the secretion of proinflammatory cytokines. Importantly,
IL-12, a cytokine necessary for Th1 polarization, was significantly greater compared to control DCs, and IL-10, which generates a Th2 response, was not activated. This is in contrast to the TLR4 agonist LPS, which upregulates secretion of IL-10. Thus, the choice of TLR agonist used for a specific TLR is also critical in study design. These results demonstrate that TLR4 activation by HBHA is important in the E.G7 thymoma model, and that both MyD88 and TRIF arms were necessary. In support of this result, another study by Narayanan et al., utilizing an E.G7-OVA lymphoma model, showed that MyD88 and CD40 signaling were required for DC-dependent antitumor activity [36].

The adoptively transferred cell type may play a significant role in the type of antitumor immunity that is activated, which may nevertheless be enhanced by TLR stimulation. Goldstein et al. recently studied whether malignant H11 lymphoma B cells treated with the TLR9 agonist $\mathrm{CpG}$ ex vivo were capable of loading antigen and activating a tumor directed CTL response [37]. TLR9 stimulated tumor B cells were then administered to mice and shown to induce antitumor $\mathrm{T}$ cell immunity; however, the major cell type activated was CD4+ T cells and not CD8+ CTL. H11 tumor cell specific immunity was observed in mice receiving $\mathrm{H} 11$ tumor cells with $\mathrm{CpG}$, whereas previous attempts to activate APCs with B-cell lymphoma tumor antigens, although capable of antigen presentation, resulted in $\mathrm{T}$ cell tolerance. Therefore, despite a predominantly CD4+ T cell response, in the presence of the TLR agonist CpG, the tumor was targeted by the host. Unfortunately, characterization of whether the CD4+ T cells responsible for tumor rejection were Th1 was not explored. Finally, the authors suggest that since TLR9 expression is not required in the tumor cell itself (or in other words TLR9 expression in healthy cells is necessary for antitumor immunity), that this approach may be applicable to other tumor types. This research is the focus of a new clinical trial for patients with mantle cell lymphoma (NCT00490529).

Emerging evidence points to the possibility that TLR stimulation may also be important in T cells [38]. Specifically, TLR8 stimulation may reverse Treg function, which naturally suppresses CTL immunity and promotes tolerance. In contrast, stimulation of other TLRs on Tregs may enhance their suppressive activity, clearly signifying that targeted TLR therapy or Treg depletion is critical for success. Treg depletion studies are currently underway in several studies; however, the major drawback to this approach could be autoimmune side-effects [39]. Upregulating TLR expression in naïve T cells is necessary for optimal T cell responses and their survival, and possibly enhances differentiation into memory cells with long-standing anti-tumor capabilities. IL-2, IL-7, IL-12, IL-15, and IL-21 are cytokines necessary to obtain T cell antitumor functionality. Thus, discovery of TLR agonists that increase expression of cytokines beneficial for $\mathrm{T}$ cell antitumor immunity is imperative. In mice with lung carcinoma, leukemia or melanoma, TLR1/2 activation by bacterial lipoprotein resulted in regression of 3LL tumors with the generation of long-term immunity against tumor challenge [40]. TLR1/2 activation was associated with inhibited Treg cell function and increased tumor-specific CTL and was not seen in SCID mice lacking T cells, suggesting that CTL responses were dependent on TLR stimulation. Thus, TLR signaling has the potential to both induce good $\mathrm{T}$ cell responses in the form of TAA-specific CTLs while simultaneously breaking tolerance.

Synthetic approaches have been undertaken to mimic the natural abilities of TAAs to stimulate T-helper responses and TLR stimulation to efficiently enhance antigen presentation on dendritic cells. Second generation liposomal vehicles containing both CTL- and CD4+ Th1specific peptide epitopes derived from ErbB2 and TLR2/1 or TLR2/6 
agonists are capable of eradicating ErbB2-expressing tumors in vivo [41]. This work suggests that a combination of TAA with TLR agonists in a synthetic delivery mechanism may enhance immunogenicity and promote long-term immunity since immunization resulted in rejection of inoculated ErbB2-negative RenCa cell tumors.

TLR stimulation may not be advantageous in all settings. Pam2 lipopeptides (TLR2/6 agonists) administered in treatment of B16 melanoma in vivo resulted in increased Treg (Foxp3+CD4+) cells [42]. This correlated with better tumor responses when Tregs were depleted with anti-CD25 antibody. Thus, TLR ligands must be carefully selected to induce Th1 polarization, tumor-specific CTLs, and longterm immunity without Treg induction. Alternatively, combination regimens with Treg depleting antibodies could enhance clinical outcomes [39].

Several clinical studies involving TLR stimulation in vivo have confirmed that TLRs are critical for effective cancer immunity. TLR agonists in combination with radiotherapy may have the potential to induce antitumor clinical responses with long term immunization [43]. Low-grade B cell lymphoma patients treated with 4Gy radiotherapy in combination with CpG-enriched PF-3512676 in situ resulted in a complete clinical response and several partial responses [44]. This strategy was successful at generating tumor-specific memory CTLs. The major advantage to this therapy design is the lack of need for an actual vaccine, however, systemic administration of TLR agonists may not be practical or meet the same results. This study suggests that TLR stimulation alone may break immunological tolerance and render lymphoma cells susceptible to immune surveillance. Moreover, patients with higher Treg induction generally performed poorer. Follow-up studies investigating the role of Treg cells as markers of poor outcome or whether they indicate potential for treatment success are ongoing.

In a completed phase I study of CDX-1307 (Celldex Therapeutics, Inc), a vaccine candidate composed of human chorionic gonadotropin $\beta$-chain fused to mannose receptor-specific antibody, administered with TLR3 and TLR7/8 agonists in patients with advanced breast, colorectal, pancreatic, ovarian, or bladder cancer, TLR stimulation enhanced antibody and T-cell specific responses [45]. This correlated with longer stable disease and clinical benefit as two patients with higher antibody titers and T-cell immunity had the best outcome. TLR stimulation was required for detection of T-cell responses to CDX1307, suggesting that TLR activation enabled development of TAAspecific responses. Similarly, MUC1 antigen is a membrane-bound TAA overexpressed on tumor cells [46]. Using Cu+-catalyzed click chemistry, TLR2 ligand PamCSK4 was conjugated to MUC1 tandem repeats in mono-, di- and trivalent fashion. This approach uses TLR agonists in combination with TAA much like CDX-1307. The ability of this technology to activate antitumor immunity is currently under preclinical evaluation.

Recently, a phase I/II trial was initiated to study the effect of Ampligen (poly-I:poly-C12U), a TLR3 agonist, in combination with oxidized tumor lystate (OC-L) as a vaccine against recurrent ovarian, fallopian tube or primary peritoneal cancer (NCT01312389). This design may be effective in generating antitumor immune responses to multiple TAAs since the vaccine utilizes whole tumor lysates.

Patients with advanced or metastatic melanoma have a 5-year survival rate of less than $10 \%$. A large phase III trial was recently completed using gp100:209-217 (210M) peptide vaccination with or without IL-2 administration [47]. Patients receiving the vaccine with IL-2 showed a $16 \%$ overall clinical response and median survival of 17.8 months versus $6 \%$ and 11.1 month survival for those patients receiving only IL-2, respectively. And while the study design was to assess the contribution of vaccine versus IL-2 alone, the vaccine enhanced cytokine therapy and presumably enhanced CD8+ T cell responses. The peptide vaccine was complexed in incomplete Freund's adjuvant (Montanide ISA-51), which may induce a TLR-dependent response. Moreover, Amos et al. recently demonstrated that gp100 (25-33)-specific T cell activity is enhanced upon TLR3 and TLR9 stimulation, which enabled tumor recognition through enhanced immunogenicity [16]. Perhaps future studies will incorporate TLR-specific agonists that may augment vaccine and cytokine therapy for advanced melanoma and other cancers. Currently, a phase II clinical trial aimed at investigating the therapeutic effectiveness of the TLR8 agonist, resiquimod, is being studied in another gp100/melanoma antigen encoded gene (MAGE) vaccination protocol (NCT00960752). This protocol also will look specifically at the contribution of plasmacytoid DCs (pDC) to innate immune activation at the vaccination site. In the Netherlands, a phase I/II study is also investigating the toxicity and clinical efficacy of a TLRmatured DC vaccine against advanced melanoma (NCT00940004).

The glioblastoma survival rate at 5 years is less than 3.3\% [48]. Previously in a phase I trial, it was found that patients treated with an autologous DC vaccine pulsed with tumor peptides resulted in low toxicity and antitumor CTL responses with one clinical response. Despite poor clinical activity, the stimulatory activity of the vaccine was effective. Therefore, a phase II trial designed to test the DC cancer vaccine against WHO grade III or IV glioma is being investigated in combination with either TLR3 agonist poly ICLC or TLR7 agonist imiquimod (NCT01204684) to enhance clinical efficacy. The TLR ligands will hopefully provide an adjuvant effect, enhancing recognition of the tumor cells by the host immune system.

\section{Actions of TLR ligands on pDCs may be critical for anti- tumor vaccination}

pDCs are a critical immune effector cell involved in arming of the innate and adaptive immune responses. pDCs express only TLR7 and TLR9 and via stimulation of these pathways are the chief interferon producing cells in the body in response to viral infection. Multiple lines of evidence have shown that pDCs are capable of antigen capture and presentation [49-51]. Ligation/activation of TLR7 and 9 via synthetic ligands or viral infection induces production of type I interferon and cytokines from pDCs which contribute to the ability of pDCs to both prime and boost primarily T cells [50-52] and also to activate NK cells $[53,54]$. The ability of pDCs to activate both T cells and NK cells in response to TLR agonists makes them an intriguing target for possible immunotherapeutic approaches to cancer treatment. At this point only a few studies have progressed far enough to begin to show the clinical relevance of pDCs as a component of a possible cancer vaccine strategy.

In 2007, stage I/II melanoma patients were injected intradermally in the sentinel lymph node (SLN) with a soluble CpG-B ODN to determine whether pDC activation could lead to possible tumor suppression [55]. Their findings indicate that injection of CpG-B does indeed activate pDCs, based on expression of the surface markers CD86 and CD40. In addition they also noted that injection of CpG-B resulted in increased leukocytes in the SLN, increased release of inflammatory cytokines, and lower numbers of suppressive $\mathrm{T}$ regulatory cells. In support of these observations, recent work by Nierkins et al., also indicates that pDCs and TLR stimulation can be critical in establishing an anti-tumor environment, and that stimulation of TLR9 is required for this effect. They showed that the introduction of wild type pDCs alone into TLR9 -/- mice restored the ability to cross-prime both antigen-specific and functional CTLs upon CpG stimulation. They also showed that pDCs 
were critical for the maturation of conventional DCs, the upregulation of CD80, and the ability of the conventional DCs (cDCs) to crosspresent antigen [56]. These results are extremely promising and indicate that $\mathrm{pDCs}$ and TLR stimulation may be necessary for effective CTL responses in the context of cancer immunity.

Additionally, the therapeutic importance of pDCs was the focus of a novel cancer vaccine strategy [57]. This study employed HLA matched allogeneic pDCs pulsed with TAAs to test the ability of these pDCs to induce tumor specific immune responses. In melanoma patients injected with TAA-loaded pDCs, CTLs were able to kill autologous tumor cells. In comparison to a similar strategy using myeloid DCs (mDCs), the pDCs elicited a much stronger induction of tumor specific CTLs with increased function as well. In addition, the CTLs created via the pDCs were much more efficient in the killing of autologous tumor cells than CTLs generated from mDCs. These results are very exciting and support the notion that $\mathrm{pDCs}$ can be a critical component of a candidate cancer vaccine strategy. It would be interesting to determine if the anti-tumor response would be even more profound if the peptide-pulsed pDCs were simultaneously activated with TLR7 or TLR9 agonists.

\section{Conclusions}

TLR combination therapy regimens for cancer vaccines hold significant promise for enhancing the efficacy of immunotherapeutics. Activation of innate and adaptive immune responses, blocking inhibitory cells and possibly generating long-term immunity against cancer remission are just a few of the possibilities. In the past year, exciting clinical data has emerged demonstrating that TLRs promote tumor immunity against some of the most common and deadly cancers. As exploring the addition of TLR ligands to these regimens increases, we must be cautious and choose the specific TLR agonist that works best for a particular protocol. The incorrect choice could promote autoimmune side-effects through dysregulation of tolerance or inhibit the cancer vaccine itself through the upregulation of immunosilencing factors. In addition, immunotherapeutics are costly and take a considerable amount of time to generate. Even though these therapies may be highly effective, they may lose practicality if costs balloon, insurance companies choose not to cover the drug or clinics are not swiftly reimbursed for large outlays, as was recently observed by the negative publicity received by Dendreon, the makers of Sipuleucel-T. Still, immunotherapy is the next frontier in personalized medicine and efforts to make it more effective through the coadminstration of TLR ligands are exciting and highly promising, offering new therapeutics that literally bring the bench to the bedside with one treatment.

\section{Acknowledgements}

BD is supported by NIH grants CA096500, DE018281, and a Burroughs Wellcome Fund grant. SMG is supported by NIH training grant T32-AI007001. BD is a Leukemia \& Lymphoma Society Scholar and Burroughs Wellcome Fund Investigator in Infectious Disease.

\section{References}

1. Shankaran V, H Ikeda, Bruce AT, White JM, Swanson PE, et al. (2001) IFNgamma and lymphocytes prevent primary tumour development and shape tumour immunogenicity. Nature 410: 1107-1111.

2. Wang RF, Peng G, Wang HY (2006) Regulatory T cells and Toll-like receptors in tumor immunity. Semin Immunol 18: 136-142.

3. Gattinoni L, Powell DJ Jr., Rosenberg SA, Restifo NP (2006) Adoptive immunotherapy for cancer: building on success. Nat Rev Immunol 6: 383-393.

4. Heslop HE, Rooney CM (1997) Adoptive cellular immunotherapy for EBV lymphoproliferative disease. Immunol Rev 157: 217-222.
5. Perret R, Ronchese F (2008) Memory T cells in cancer immunotherapy: which CD8 T-cell population provides the best protection against tumours? Tissue Antigens 72: 187-194.

6. Bendandi M, Gocke CD, Kobrin CB, Benko FA Sternas LA et al. (1999) Complete molecular remissions induced by patient-specific vaccination plus granulocyte-monocyte colony-stimulating factor against lymphoma. Nat Med 5: 1171-1177.

7. Kwak LW, Campbell MJ, Czerwinski DK, Hart S, Miller RA et al. (1992) Induction of immune responses in patients with B-cell lymphoma against the surface-immunoglobulin idiotype expressed by their tumors. N Engl J Med 327 1209-1215.

8. Neelapu SS, LW Kwak, CB Kobrin, CW Reynolds, JE Janik, et al. (2005) Vaccine-induced tumor-specific immunity despite severe B-cell depletion in mantle cell lymphoma. Nat Med 11: 986-991.

9. Shore ND (2011) Conference scene: Phacilitate barcelona active immunotherapeutics forum. Immunotherapy 3: 931-932.

10. Kantoff PW, Higano CS, Shore ND, Berger ER, Small EJ, et al. (2010) Sipuleucel-T immunotherapy for castration-resistant prostate cancer. N Eng J Med 363: 411-422.

11. Kantoff PW, Schuetz TJ, Blumenstein BA, Glode LM, Bilhartz DL, et al. (2010) Overall survival analysis of a phase II randomized controlled trial of a Poxviralbased PSA-targeted immunotherapy in metastatic castration-resistant prostate cancer. J Clin Oncol 28: 1099-1105.

12. Lassi K, and Dawson NA (2009) Emerging therapies in castrate-resistant prostate cancer. Curr Opin Oncol 21: 260-265.

13. Davis MB, Vasquez-Dunddel D, Fu J, Albesiano E, Pardoll D, et al. (2011) Intratumoral administration of TLR4 agonist absorbed into a cellular vector improves antitumor responses. Clin Cancer Res 17: 3984-3992.

14. Kawai T, Akira S (2010) The role of pattern-recognition receptors in innate immunity: update on Toll-like receptors. Nat Immunol 11: 373-384

15. Duthie MS, Windish HP, Fox CB, and Reed SG (2011) Use of defined TLR ligands as adjuvants within human vaccines. Immunol Rev 239: 178-196.

16. Amos SM, Pegram HJ, Westwood JA, John LB, Devaud C, (2011) Adoptive immunotherapy combined with intratumoral TLR agonist delivery eradicates established melanoma in mice. Cancer Immunol Immunother 60: 671-683.

17. Adams S, O'Neill DW, Nonaka D, Hardin E, Chiriboga L, et al. (2008) Immunization of malignant melanoma patients with full-length NY-ESO-1 protein using TLR7 agonist imiquimod as vaccine adjuvant. J Immunol 181: 776-784.

18. Tanaka J, Sugimoto K, Shiraki K, Tameda M, Kusagawa S, et al. (2010) Functional cell surface expression of toll-like receptor 9 promotes cel proliferation and survival in human hepatocellular carcinomas. Int J Oncol 37 805-814.

19. Shen P, Jiang T, Lu H, Han H, and Luo R (2011) Combination of Poly I:C and arsenic trioxide triggers apoptosis synergistically via activation of TLR3 and mitochondrial pathways in hepatocellular carcinoma cells. Cell 35: 803-810.

20. Hemmi H, Takeuchi O, Kawai T, Kaisho T, Sato S, et al. (2000) A Toll-like receptor recognizes bacterial DNA. Nature 408: 740-745.

21. Agrawal S, Agrawal A, Doughty B, Gerwitz A, Blenis J, et al. (2003) Cutting edge: different Toll-like receptor agonists instruct dendritic cells to induce distinct Th responses via differential modulation of extracellular signal-regulated kinasemitogen-activated protein kinase and c-Fos. J Immunol 171: 4984-4989.

22. Malek TR, Castro I (2010) Interleukin-2 receptor signaling: at the interface between tolerance and immunity. Immunity $33: 153-165$.

23. Salaun B, Lebecque S, Matikainen S, Rimoldi D, Romero P (2007) Toll-like receptor 3 expressed by melanoma cells as a target for therapy? Clin Cancer Res 13: 4565-4574.

24. Salaun B, Coste I,. Rissoan MC, Lebecque SJ, Renno T (2006) TLR3 can directly trigger apoptosis in human cancer cells. J Immunol. 176: 4894-4901.

25. Brignole C, Marimpietri D, Di Paolo D, Perri P, Morandi F, et al. (2010) Therapeutic targeting of TLR9 inhibits cell growth and induces apoptosis in neuroblastoma. Cancer Res 70: 9816-9826

26. Melief CJ, Van Der Burg SH, Toes RE, Ossendorp F, and Offringa R (2002) 
Citation: Gregory SM, West JA, Damania B (2011) Cancer Vaccination, Will You Have To Pay The Toll? J Vaccines Vaccin S1:001. doi:10.4172/21577560.S1-001

Effective therapeutic anticancer vaccines based on precision guiding of cytolytic T lymphocytes. Immunol Rev 188: 177-182.

27. Klebanoff CA, Acquavella N, Yu Z, Restifo NP (2011) Therapeutic cancer vaccines: are we there yet? Immunol Rev 239: 27-44.

28. Apetoh L, Locher C, Ghiringhelli F, Kroemer G, and Zitvogel L (2011) Harnessing dendritic cells in cancer. Semin Immunol 23: 42-49.

29. Conroy H, Marshall NA, Mills KH (2008) TLR ligand suppression or enhancement of Treg cells? A double-edged sword in immunity to tumours. Oncogene 27: 168-180.

30. Pasare C, Medzhitov R (2003) Toll pathway-dependent blockade of CD4+CD25+ T cell-mediated suppression by dendritic cells. Science 299 : 1033-1036.

31. Yang Y, Huang CT, Huang X, Pardoll DM (2004) Persistent Toll-like receptor signals are required for reversal of regulatory $T$ cell-mediated CD8 tolerance. Nat Immunol 5: 508-515.

32. Peng G, Guo Z, Kiniwa Y, Voo KS, Peng W, et al. (2005) Toll-like receptor 8-mediated reversal of CD4+ regulatory $T$ cell function. Science 309: 13801384.

33. Fotin-Mleczek M, Duchardt KM, Lorenz C, Pfeiffer R, Ojkic-Zrna S, et al (2011) Messenger RNA-based vaccines with dual activity induce balanced TLR-7 dependent adaptive immune responses and provide antitumor activity. $\mathrm{J}$ Immunother 34: 1-15.

34. Lin Y, Zhang L, Cai AX, Lee M, Zhang W, et al. (2011) Effective posttransplan antitumor immunity is associated with TLR-stimulating nucleic acidimmunoglobulin complexes in humans. J Clin Invest 121: 1574-1584.

35. Jung ID, SK Jeong, CM Lee, KT Noh, DR Heo, YK et al. (2011) Enhanced efficacy of therapeutic cancer vaccines produced by co-treatment with Mycobacterium tuberculosis heparin-binding hemagglutinin, a novel TLR4 agonist. Cancer 71: 2858-2870.

36. Narayanan P, Lapteva N, Seethammagari M, Levitt JM, Slawin KM, et al. (2011) A composite MyD88/CD40 switch synergistically activates mouse and human dendritic cells for enhanced antitumor efficacy. J Clin Invest 121: 1524-1534.

37. Goldstein MJ, Varghese B, Brody JD, Rajapaksa R, Kohrt H, et al. (2011) A CpG-loaded tumor cell vaccine induces antitumor CD4+ $T$ cells that are effective in adoptive therapy for large and established tumors. Blood 117: 118127

38. Salem ML (2011) Triggering of toll-like receptor signaling pathways in T cells contributes to the anti-tumor efficacy of T cell responses. Immunol Lett 137 $9-14$.

39. Mougiakakos D, Choudhury A, Lladser A, Kiessling R, Johansson CC (2010) Regulatory T cells in cancer. AdvCancer Res 107: 57-117

40. Zhang Y, Luo F, Cai Y, Liu N, Wang L, et al. (2011) TLR1/TLR2 agonist induces tumor regression by reciprocal modulation of effector and regulatory $T$ cells. J Immunol 186: 1963-1969.

41. Thomann JS, Heurtault B, Weidner S, Braye M, Beyrath J, et al. (2011) Antitumor activity of liposomal ErbB2/HER2 epitope peptide-based vaccine constructs incorporating TLR agonists and mannose receptor targeting. Biomaterials 32: 4574-4583.

42. Yamazaki S, Okada K, Maruyama A, Matsumoto M, Yagita $H$ et al (2011) TLR2-dependent induction of IL-10 and Foxp3+ CD25+ CD4+ regulatory T cells prevents effective anti-tumor immunity induced by Pam2 lipopeptides in vivo. PLoS One 6: e18833.

43. Li R, Song Y, Chen W (2011) Enhancing radiosensitivity of human pulmonary adenocarcinoma cell line A549 by CpG ODN1826. Cancer 26: 69-76.

44. Brody JD, Ai WZ, Czerwinski DK, Torchia JA, M Levy, et al. (2010) In situ vaccination with a TLR9 agonist induces systemic lymphoma regression: a phase I/II study. J Clin Oncol 28: 4324-4332.
45. Morse MA, Chapman R, Powderly J, Blackwell K, Keler T et al (2011) Phase Study Utilizing a Novel Antigen-Presenting Cell-Targeted Vaccine with Toll-like Receptor Stimulation to Induce Immunity to Self-antigens in Cancer Patients. Clin Cancer Res 17: 4844-4853.

46. Cai H, Huang ZH, Shi L, Zhao YF, Kunz H et al. (2011) Towards a fully synthetic MUC1-based anticancer vaccine: efficient conjugation of glycopeptides with mono-, di-, and tetravalent lipopeptides using click chemistry. Chemistry 17 6396-6406.

47. Schwartzentruber DJ, Lawson DH, Richards JM, Conry RM, Miller DM, et al. (2011) gp100 peptide vaccine and interleukin-2 in patients with advanced melanoma. N Engl J Med 364: 2119-2127.

48. Deorah S, Lynch CF, Sibenaller ZA, Ryken TC (2006) Trends in brain cance incidence and survival in the United States: Surveillance, Epidemiology, and End Results Program, 1973 to 2001. Neurosurg Focus 20:E1.

49. Hoeffel G, Ripoche AC, Matheoud D, Nascimbeni M, Escriou N, et al. (2007) Antigen crosspresentation by human plasmacytoid dendritic cells. Immunity 27 : 481-492.

50. Sapoznikov A, Fischer JA, Zaft T, Krauthgamer R, Dzionek A, et al. (2007) Organ-dependent in vivo priming of naive CD4+, but not CD8+, $T$ cells by plasmacytoid dendritic cells. J Exp Med 204: 1923-1933.

51. Mouries J, Moron G, Schlecht G, Escriou N, Dadaglio G, et al. (2008) Plasmacytoid dendritic cells efficiently cross-prime naive $T$ cells in vivo after TLR activation. Blood 112: 3713-3722.

52. Schlecht G, Garcia S, Escriou N, Freitas AA, Leclerc C, et al (2004) Murine plasmacytoid dendritic cells induce effector/memory CD8+ T-cell responses in vivo after viral stimulation. Blood 104: 1808-1815.

53. Liu C, Lou Y, Lizee G, Qin H, Liu S, et al. (2008) Plasmacytoid dendritic cells induce NK cell-dependent, tumor antigen-specific T cell cross-priming and tumor regression in mice. J Clin Invest 118: 1165-1175.

54. Kalinski P Mailliard RB, Giermasz A, Zeh HJ, Basse P, et al. (2005) Natura killer-dendritic cell cross-talk in cancer immunotherapy. Expert Opin Biol The 5: 1303-1315.

55. Molenkamp BG, van Leeuwen PA, Meijer S, Sluijter BJ, Wijnands PG, et al. (2007) Intradermal CpG-B activates both plasmacytoid and myeloid dendritic cells in the sentinel lymph node of melanoma patients. Clin Cancer Res 13 2961-2969.

56. Nierkens S, den Brok MH, Garcia Z, Togher S, Wagenaars J, et al. (2011) Immune adjuvant efficacy of $\mathrm{CpG}$ oligonucleotide in cancer treatment is founded specifically upon TLR9 function in plasmacytoid dendritic cells. Cancer Res 71: 6428-6437.

57. Aspord C, Charles J, Leccia MT, Laurin D, Richard MJ, et al. (2010) A novel cancer vaccine strategy based on HLA-A*0201 matched allogeneic plasmacytoid dendritic cells. PLoS One 5:e10458.
This article was originally published in a special issue, Tumor Immunology Vaccines handled by Editor(s). Dr. Robert John Amato, The University of Texas, USA 